\title{
A database of polarimetric and multispectral images in the visible and NIR regions
}

\author{
Pierre-Jean Lapray ${ }^{\mathrm{a}}$, Luc Gendre ${ }^{\mathrm{a}}$, Alban Foulonneau ${ }^{\mathrm{a}}$, and Laurent Biguéa \\ aRIMAS, EA7499, Université de Haute-Alsace, Mulhouse, France
}

\begin{abstract}
Multi-band polarization imaging, by mean of analyzing spectral and polarimetric data simultaneously, is a good way to improve the quantity and quality of information recovered from a scene. Therefore, it can enhance computer vision algorithms as it permits to recover more statistical information about a surface than color imaging. This work presents a database of polarimetric and multispectral images that combine visible and near-infrared (NIR) information. An experimental setup is built around a dual-sensor camera. Multispectral images are reconstructed from the dual-RGB method. The polarimetric feature is achieved using rotating linear polarization filters in front of the camera at four different angles $(0,45,90$ and 135 degrees). The resulting imaging system outputs 6 spectral/polarimetric channels. We demonstrate 10 different scenes composed of several materials like color checker, high reflecting metallic object, plastic, painting, liquid, fabric and food. Our database of images is provided online as supplementary material for further simulation and data analysis. This work also discusses several issues about the multi-band imaging technique described.
\end{abstract}

Keywords: Polarimetric imaging, spectral imaging, image database

\section{INTRODUCTION}

Polarimetry is the analysis of the wave oscillation of the electric field of light beams. The light could be unpolarized or polarized (fully or partially) under a given direction. Several animals like fishes, insects, reptiles or birds are capable to perceive polarized light, ${ }^{1}$ whereas humans can not. Thanks to specific photoreceptors, the polarized light vision helps the survival of animals by reacting according to their light environment. Additionally, some animals need accurate color vision, e.g. the honeybees to search for flowers as nectar or pollen sources. Polarimetric imaging techniques use this polarization phenomenon, for example, in computer vision applications like specular and diffuse separation, ${ }^{2}$ target detection, ${ }^{3}$ shape estimation ${ }^{4}$ or material classification. ${ }^{5}$

Multispectral imaging has been found to be of great interest in the last few decades. It is a mode commonly reported in the literature for the enhancement of the color reproduction, ${ }^{6}$ background subtraction, ${ }^{7}$ shadow detection ${ }^{8}$ or illuminant estimation. ${ }^{9}$ In most of natural scenes, even in presence of manufactured objects, reflectance spectra are relatively smooth functions across wavelengths. Nevertheless, three-channel camera appears to be insufficient to estimate accurately the spectral reflectance of a surface: there are several different spectra power distributions that could correspond to the same RGB digital camera values. Thus, spectral ambiguity can be reduced by increasing the number of spectral channels. It has been found that spectral reconstruction images using few bands could have a performance close to that of hyperspectral imagery, but with higher spatial resolution, lower acquisition time, cost and complexity. ${ }^{10}$ In the literature, the discrimination between hyperspectral and multispectral imaging systems seems to be not well defined. The difference lies mainly in the number of bands used to capture the information distributed in a given wavelength range. Thus, multispectral imaging has generally less spectral sensors than hyperspectral for the same sensed spectrum, with wider band sensitivities. But the band-pass filter design with extremely narrow or wide responses per band could not reach to a satisfactory result in term of spectral reconstruction. ${ }^{11}$ Typically, examples in the literature

Further author information: (Send correspondence to Pierre-Jean Lapray)

E-mail: pierre-jean.lapray@uha.fr 
show that multispectral imaging in the visible could be achieved by using six channels with good spectral estimation. ${ }^{12}$

Methods for multispectral imaging in the visible domain could also be extended to near-infrared spectrum. Measuring intensities in both the visible and Near-InfraRed (NIR) spectra has gained in interest in recent years. NIR light is material dependent. For example, several paints with the same green colorization pigment, but composed by different binding agents, will reflect differently NIR light. NIR light can also penetrate deeper in materials than visible wavelength. New emerging imaging applications exploit the joint acquisition of visible and NIR for image dehazing, ${ }^{13}$ semantic segmentation, ${ }^{14}$ plant analysis or image denoising. ${ }^{15}$

Recently, mixing spectral and polarimetric information into a multi-band bio-inspired acquisition system has become an emerging research subject. ${ }^{16-19}$ Some recent advances in designing new sensor technologies enable the acquisition of spectropolarimetric information in a snapshot way. ${ }^{20}$ Like for Bayer sensors, micro-polarizer filters can be attached directly in front of the pixel array, so that each photo-diode senses a different direction of the linearly polarized incoming light. Few works exploit the correlations between reflectance and degree of polarization of a polarized light beam reflected onto a surface. ${ }^{21}$ It appears that the spectral and polarimetric signatures of objects seem correlated.

We thought that data coming from spectropolarimetric imaging systems are still under-exploited, sample of multi-band images are not available, and dedicated imaging pipeline is still an open subject.

This paper tries to deal with these statements. The contributions of this work are:

- A practical imaging pipeline, including a calibration procedure, and recovering of spatial, spectral and polarimetric data from a set of photographs,

- A database of multispectral and polarimetric images of various materials in the visible and nearinfrared part of the spectrum,

- Analysis and discussion of joint reflectance and polarimetric estimations.

We begin the paper by the imaging model definition in Section 2. We then present, in Section 3, a laboratory setup and an imaging pipeline for recovering images that span the visible and near-infrared. We then describe the captured image database in Section 4, before to conclude in Section 5.

\section{IMAGING MODEL}

Most of the imaging algorithms, like spectral reconstruction or Stokes parameter estimation, consider linear optical models plus an additive noise model to characterize the detector used. In the following section, we will describe the linear models for both spectral and polarimetric imaging.

\subsection{Spectral reflectance estimation}

The output camera responses are relative to the integration of all the energy that reaches the sensor weighted by the sensitivities of each camera band. Optical elements (i.e. the bandpass and polarimetric filter transmissions) participate to the global camera sensitivity. Assuming already noise-corrected camera responses, the camera response model spanning the visible and near-infrared wavelengths can be described by the following equation:

$$
\rho_{i}=\int_{\lambda=420}^{\lambda=1000} E(\lambda) R(\lambda) O(\lambda) C_{i}(\lambda) d \lambda,
$$

where $\rho_{i}$ is the camera response of the $i$ 'th channel, $E(\lambda)$ is the spectral emission of the illuminant, $R(\lambda)$ is the reflectance, $O(\lambda)$ is the global transmittance of all the optical elements and $C_{i}(\lambda)$ is the spectral sensitivity of the $i$ 'th channel. 
We now assume that the spectral functions are sampled at $n$ points with the same interval $\Delta \lambda$ in the range $[420,1000 \mathrm{~nm}]$. The previous relation becomes:

$$
\rho_{i}=\sum_{\lambda=420}^{\lambda=1000} E(\lambda) R(\lambda) O(\lambda) C_{i}(\lambda)
$$

The spectral behavior of elements from the factor $E(\lambda) O(\lambda) C_{i}(\lambda)$ are often partially or inaccurately known, or some of them may change over time. We make the assumption that spectral radiance of the illuminant and optical transmittance are fixed, so we merge all the terms into a $k \times n$ sensor matrix that we call $\mathbf{M}$, where $k$ is the number of channels of the imaging system. Let $\mathbf{R}$ be the $n \times 1$ spectral reflectance factors of the surface, and $\rho$ a $k \times 1$ matrix of sensor responses, we can simplify the model and summarize the relationship into a matrix scalar product as follows:

$$
\rho=\mathrm{MR}
$$

where $\mathbf{M}$ is the spectral measurement matrix, including the camera spectral sensitivity weighted by the illuminant power distribution and the optic transmittance effects. It then defines $k$ linear equations with $n$ unknowns.

Reflectance reconstruction is an inverse problem. Multiple algorithms exist to perform this task, like

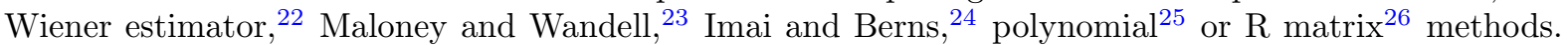
Some of the methods are specific to noise level, spectral power distribution of illuminant or require accurate spectral sensitivities of the camera. We want to remain as general as possible, and as our acquisition system has no accurate characterization data for camera sensitivities and spectral power distribution of illuminant (not completely provided by the manufacturer), we choose a simple linearmodel approach. Reflectance reconstruction from multispectral images is valid if the same illumination is used for system calibration and image acquisition. After determining $\boldsymbol{M}$ (calibration procedure will be described in Section 3.3), any given spectral reflectance could be estimated from camera responses using this equation:

$$
\hat{\boldsymbol{R}}=\mathbf{M}^{-1} \rho
$$

To recover reflectance data from measurements, with sampled data at each $10 \mathrm{~nm}$ in the range of $[420,1000 \mathrm{~nm}]$, we typically need 59 sensors. But for reflectance recovery using multispectral acquisition system, state-of-the-art shows that a smaller number of sensors could be used. ${ }^{12}$ The practical implementation in this paper will define a 6-band acquisition system in the Section 3.

\subsection{Stokes estimation}

Linear Stokes vectors are derived from irradiance measurements. They are measured by rotating a linear polarizer in front of a radiometer. In case of ideal polarizing elements, the detected light intensities are generally expressed by:

$$
\boldsymbol{I}=\mathbf{A S}
$$

where $\boldsymbol{S}$ is the input Stokes vector, $\boldsymbol{I}$ is the vector containing camera responses recovered from a set of images taken using a linear polarizer, usually oriented at four specific angles $0^{\circ}, 45^{\circ}, 90^{\circ}$ and $135^{\circ}$. The vector thus contains four light intensities such as $\boldsymbol{I}=\left[\boldsymbol{\rho}_{\mathbf{0}}, \boldsymbol{\rho}_{\mathbf{4 5}}, \boldsymbol{\rho}_{\mathbf{9 0}}, \boldsymbol{\rho}_{\mathbf{1 3 5}}\right]^{T}$. A is the polarimetric measurement matrix and its ideal expression is given by:

$$
\mathbf{A}=\frac{1}{2}\left[\begin{array}{cccc}
1 & 1 & 0 & 0 \\
1 & 0 & 1 & 0 \\
1 & -1 & 0 & 0 \\
1 & 0 & -1 & 0
\end{array}\right]
$$




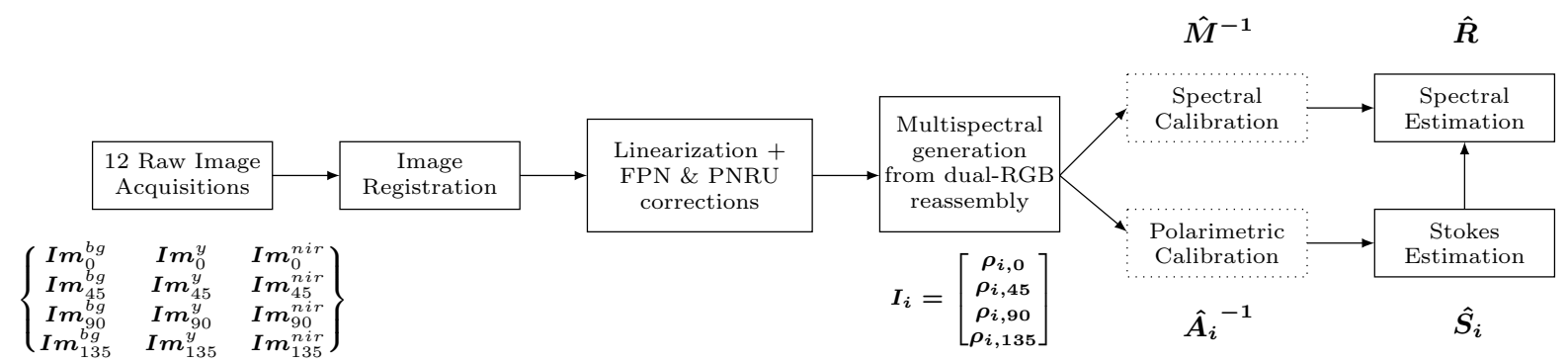

Figure 1. Practical imaging pipeline. It includes acquisition, calibration and estimation steps. Raw images are captured using linear polarizers oriented at four polarization angles, and using the dual-RGB method described in Section 3.1.

Estimation of any incident Stokes vector from camera responses can be done using the following equation:

$$
\hat{\boldsymbol{S}}=\mathbf{A}^{+} \boldsymbol{I}
$$

In real cases, deviation from ideal matrix may occur, because of diattenuation, retardance and depolarization effects of optical elements that compose the polarimeter. A calibration step is then needed to estimate the measurement matrix properly. A practical calibration procedure will be described later in Section 3.3.

The Stokes vector is function of wavelength and angle of light beam incidence. In typical cases where Stokes vector is measured using coherent illumination at a single wavelength, one simple calibration step is needed. But for broadband measurement, we have to consider independently the polarization properties of optical elements that composes the analyzer. In case of using discrete imaging elements (i.e. pixel), bandpass filters, and under a single angle of view, Stokes measurement is determined from an average of irradiance measurement over area, wavelength and solid angle. If we consider a multispectral system with relatively narrow bands, the spectral dependence of $\boldsymbol{A}$ has to be considered, so that each channel $i$ senses a different Stokes vector. ${ }^{27}$

From these statements, the Equation 7 becomes:

$$
\hat{\boldsymbol{S}}_{\boldsymbol{i}}=\left[\begin{array}{c}
\hat{s}_{0, i} \\
\hat{s}_{1, i} \\
\hat{s}_{2, i} \\
0
\end{array}\right]=\mathbf{A}_{\mathbf{i}}^{+} \boldsymbol{I}_{\boldsymbol{i}},
$$

where $\mathbf{A}_{\mathbf{i}}^{+}$is pseudo-inverse of the measurement matrix resulting from the calibration procedure over different spectral bands $i$.

\subsection{Multi-band imaging pipeline}

Our goal is to capture both spectral and polarimetric data. Starting from the previous model definitions, we propose a pipeline containing all processing steps needed. The global imaging pipeline is shown in Figure 1.

First, we start from raw images containing both spectral and polarimetric data. Spectral and polarimetric information is recovered by acquiring multiple images, using a set of optical elements, i.e. filters, moving in front of the camera. We use the dual-RGB method to produce 6-band images from an RGB recombination of images taken in front of bandpass filters (blue-green and yellow filters). ${ }^{28}$ Moreover, a linear polarization filter is used for analyzing the polarization states, using 4 polarization angles. Due to misalignment and optics effects, a registration step is then applied. Then, digital values from the camera have to be corrected for non-linearity and fixed-pattern noise (dark offset and spatial nonuniformity 
of the lighting), as it is done in intensity/color imaging. Then, spectral and polarimetric calibration steps are implemented. We will describe later the calibration steps. Finally, spectral reconstruction and polarimetric descriptors estimation are done following the models established in Section 2.1 and 2.2.

In the following section, we will describe the practical implementation of the pipeline.

\section{IMAGE ACQUISITION}

\subsection{Experimental setup}

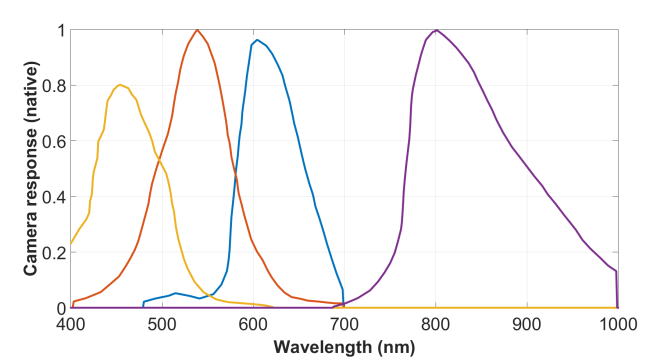

(a)

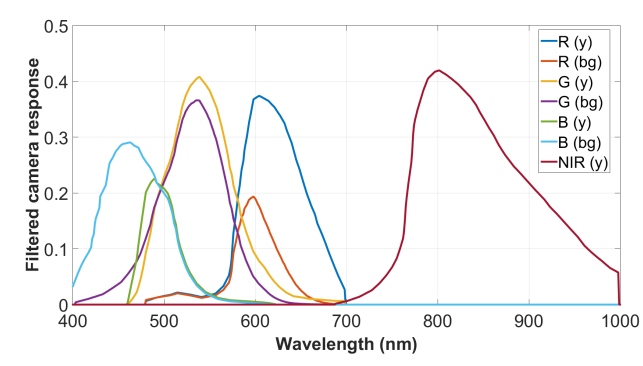

(c)

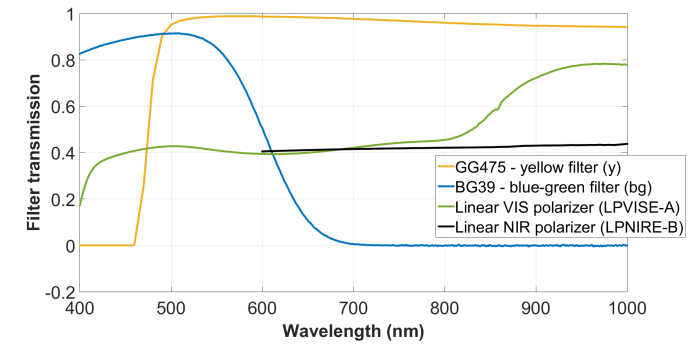

(b)

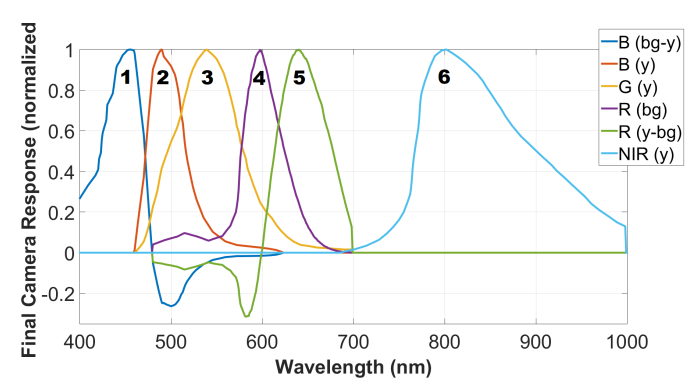

(d)

Figure 2. Dual-RGB camera response definition using the JAI camera and two absorptive filters; yellow (y) and blue-green (bg). (a) Spectral sensitivities of the JAI camera. (b) Spectral transmission of the polarimetric (for unpolarized light) and the pair of bandpass filters. The RGB inter-channel combinations in (c) define the final 6-band normalized sensitivity of our acquisition system in (d).

Acquisition setup is shown on Figure 3.

The main elements that compose the acquisition chain is a prism-based 2CCD camera (JAI AD080GE). This two-sensor system permits to simultaneously capture visible and near-infrared light with $1024 \times 768$ active pixels per channel. The quantization level is 12-bit for the NIR channel and 12-bit output for raw Bayer output.

Multispectral measurements often require complex and expensive devices. A method to design a multiple spectral band acquisition system using a conventional RGB camera was introduced by Berns et $a l^{28}$ It was originally composed by a color camera and a pair of bandpass color filters. The principle is as follows: filters are positioned one by one in front of the RGB camera, one image is taken for each filter, then RGB bands of the two images are recombined to emulate multispectral acquisition (a camera with more than 3 bands). The two bandpass filters are the blue-green BG39 (bg) and the yellow GG475 (y) filters manufactured by Schott Glass Technologies, Inc. Multispectral images $I_{i}$ are reconstructed from a set of 3 different images $\boldsymbol{I} \boldsymbol{m}^{b g}, \boldsymbol{I} \boldsymbol{m}^{y}, \boldsymbol{I m}^{\text {nir }}$. Figure 2 shows the spectral response of the system using the dual-RGB method.

Polarization data are recovered using two linear polarizing filters, one for the visible band (LPVISE100), and one for the near-infrared band (LPNIRE100). We selected two different polarization filters instead of one to get nearly-flat transmissions in the two ranges considered (visible and near-infrared). These filters 


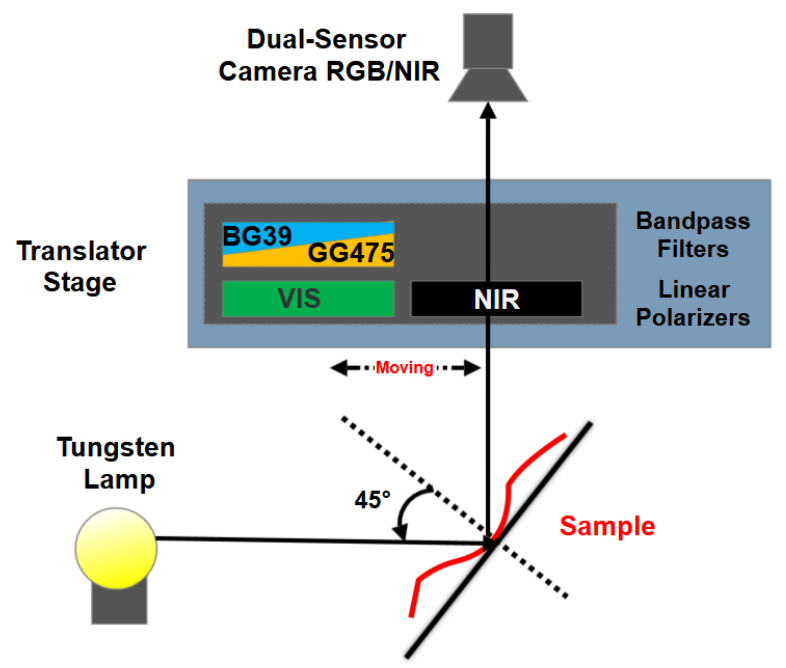

Figure 3. Experimental setup for capturing the database. The angle of incidence is set to $45^{\circ}$ to get a sufficient polarization component of light at the input of the imaging system.

are attached to two precision motorized rotation stages (Agilis ${ }^{\mathrm{TM}}$ Piezo Motor Driven Rotation Stages). A relative alignment between the two filters was done, so that both filters share the same reference angle. The stages are fixed to a translator stage to place accurately the polarizers in front of the camera. Images are taken with four polarization orientations $\left(0^{\circ}, 45^{\circ}, 90^{\circ}\right.$ and $\left.135^{\circ}\right)$. We selected two different polarization filters instead of one to get nearly-flat transmissions in the two ranges considered (visible and near-infrared).

The lighting system is a tungsten-filament lamp. This light source is positioned at approximately $100 \mathrm{~cm}$ from the target. The distance between the scene and the camera is approximately $50 \mathrm{~cm}$.

Scenes are all acquired with the same incident angle of $45^{\circ}$. It has been previously shown that a $10^{\circ}$ window around the Brewster angle is sufficient to get a significant polarization.

\subsection{Registration}

The mechanical movement of the whole set of filters (polarimetric and bandpass), along with the distortion induced by the prism inside the camera resulted in a misalignment among images. After studying this effect, it is stated that only translation differences effectively affects the images. We select the method developed by Evangelidis ${ }^{29}$ to align the image set. The Matlab toolbox is directly available in the public domain (Image Alignment Toolbox). We selected the $I m_{0}^{n i r}$ as a reference alignment image.

\subsection{Calibration}

\subsubsection{Radiometric calibration}

CMOS and CCD elements collect charges that are generally proportional to irradiance. But non-linear responses could occur due to electronic amplification, analog-to-digital conversion, saturation, etc. Moreover, many digital camera manufacturers apply some non-linearity mapping in the output pixel values to match and compensate for the inverse of non-linear display systems. As polarization descriptors are mainly derived from relative differences among intensity images, huge errors could appear in results. ${ }^{30}$ The linearity of our camera was investigated using neutral patches from the ColorChecker Classic chart, along with the method described in the literature. ${ }^{31}$ It has been found that the camera has a nearto-linear behavior, so it exists a relationship between irradiance and pixel brightness. A small gamma function is applied for each band to get the final linear responses.

Additionally, we also correct images for dark noise and spatial nonuniformity of lighting, by employing the methods from state-of-the-art. ${ }^{31}$ 


\subsubsection{Spectral calibration}

A set of known reflectance spectra are employed to recover the model parameters, i.e. the matrix $\mathbf{M}$ from Equation 3. We choose the Xrite Macbeth ColorChecker PassPort (MCCPP) as a training and testing chart. Training reflectance data $R_{\text {train }}$ are from the patches of the ColorChecker Classic chart, whereas test reflectance data are from the patches of the Creative Enhancement chart. The testing chart is used to verify the spectral estimation behavior of our system after calibration. Figure 4 shows the spectral behavior of both ColorChecker Classic and Creative Enhancement charts in the visible and near-infrared parts of the spectrum. According to the model described in Section 2, we calibrate the matrix $M$ using the pseudo-inverse method. $\mathbf{M}^{-\mathbf{1}}$ is estimated using the linear regression as follows:

$$
\hat{M}^{-1}=\mathbf{R}_{\text {train }} \rho_{\text {train }}{ }^{+},
$$

, where $\boldsymbol{\rho}^{+}$is the right pseudoinverse of $\boldsymbol{\rho}$.

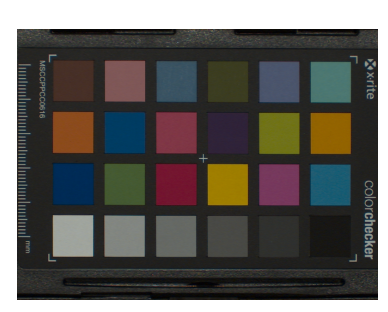

(a)

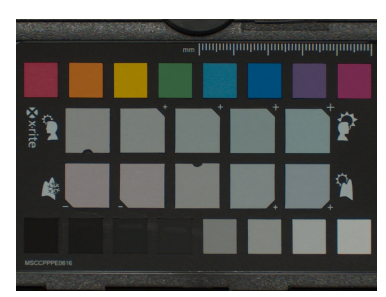

(c)

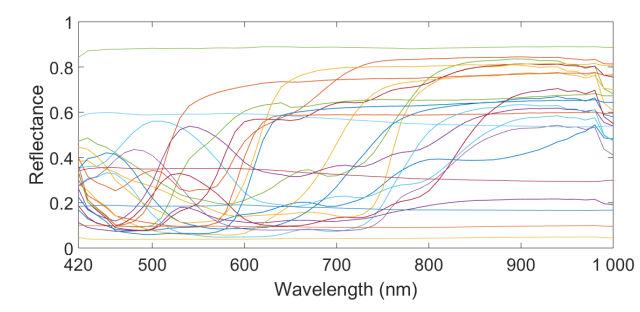

(b)

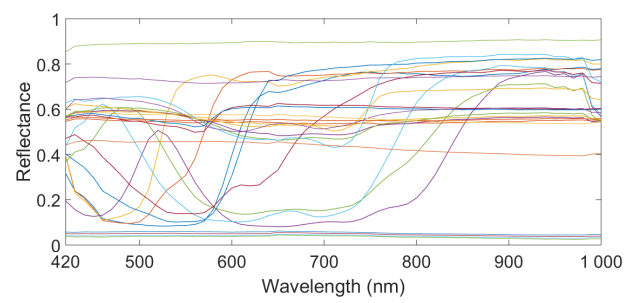

(d)

Figure 4. (a) sRGB visualization of the training target (ColorChecker Classic chart) used for spectral calibration. Color versions are obtained using a linear color transform from 6-band to XYZ, and from XYZ to sRGB. (b) Spectral reflectance of the 24 patches of the training target. ${ }^{32}$ (c) sRGB visualization of the 26 patches the testing target (Creative Enhancement Chart). (d) Spectral reflectance of the testing target. Thanks to Dr. Yusuke Monno for providing the measurement data of this chart from his work. ${ }^{33}$

\subsubsection{Polarimetric calibration}

We could summarize the calibration procedure as follows: starting from a light source (tungsten in our case), an integrating sphere is used to get unpolarized light, and a Newport 10LP-VIS-B linear polarizer is rotated to generate $Q$ reference input Stokes vectors. For each spectral band $i$ and for each generated reference Stokes vector, intensities vector $\left[\rho_{0}, \rho_{45}, \rho_{90}, \rho_{135}\right]^{T}$ is measured, leading to the following system :

$$
\boldsymbol{I}_{\boldsymbol{i}}=\left[\begin{array}{cccc}
\rho_{0, i, 1} & \rho_{0, i, 2} & \ldots & \rho_{0, i, Q} \\
\rho_{45, i, 1} & \rho_{45, i, 2} & \ldots & \rho_{45, i, Q} \\
\rho_{90, i, 1} & \rho_{90, i, 2} & \ldots & \rho_{90, i, Q} \\
\rho_{135, i, 1} & \rho_{135, i, 2} & \ldots & \rho_{135, i, Q}
\end{array}\right]=\mathbf{A}_{\mathbf{i}}\left[\begin{array}{cccc}
S_{0, i, 1} & S_{0, i, 2} & \ldots & s_{0, i, Q} \\
S_{1, i, 1} & S_{1, i, 2} & \ldots & s_{1, i, Q} \\
S_{2, i, 1} & S_{2, i, 2} & \ldots & s_{2, i, Q} \\
0 & 0 & \ldots & 0
\end{array}\right]
$$

where $\mathbf{A}$ is the $4 \times 4$ polarimetric measurement matrix to be estimated, and $s_{0-2, i}$ are the linear Stokes parameters of the incident light for each spectral band $i$. The calibration procedure with $Q=4$ is 
performed to recover an estimation of $\mathbf{A}$ for each band $i$. Thus, the ideal matrix in Equation 6 is replaced by the calibrated matrix. Once calibration is done, any input Stokes vectors could be estimated using the Equation 8 described in Section 2.

\section{DATABASE AND DISCUSSION}

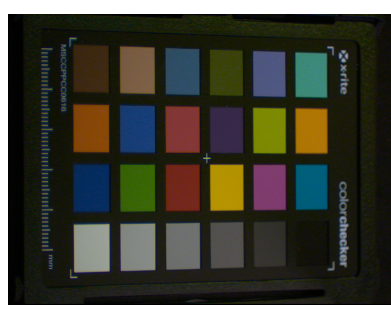

(a) ColorChecker Classic

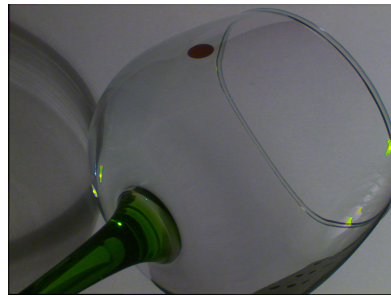

(d) Glass

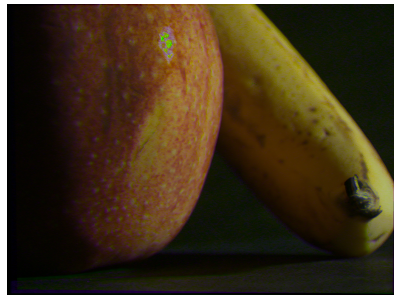

(g) Food

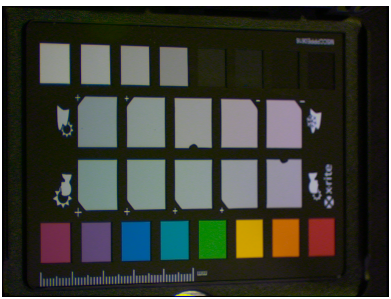

(b) ColorChecker creative enhancement

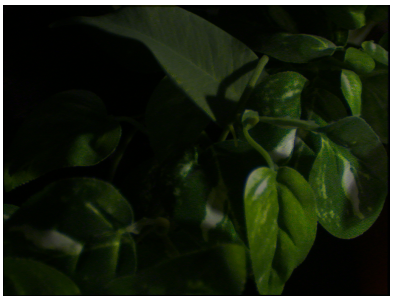

(e) Fake \& real leaves

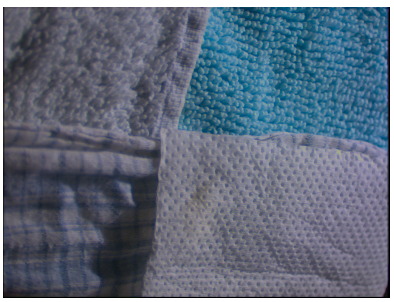

(h) Fabrics

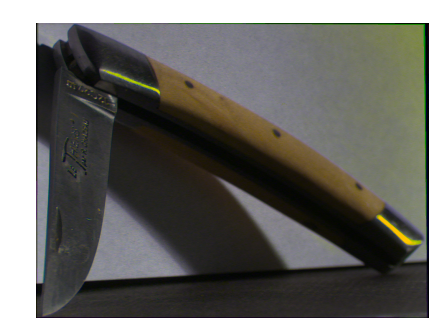

(j) Knife

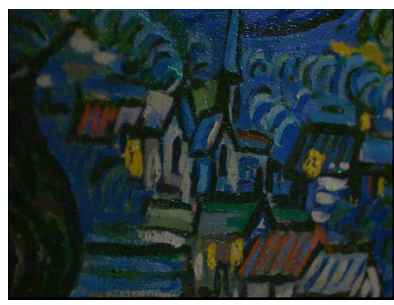

(c) Painting

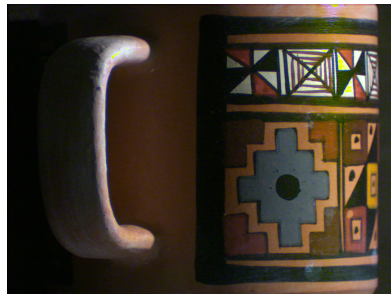

(f) Potery

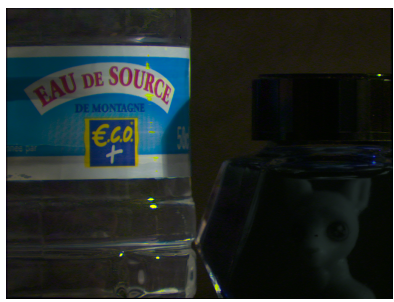

(i) Liquid

Figure 5. sRGB visualization of the 10 scenes that compose the spectral and polarimetric database. The angle of incidence is $45^{\circ}$. The database can be downloaded at https://github.com/pjlapray.

The RGB versions of the different scenes that compose the database are represented in Figure 5 . The imaging dataset is organized with the following items (and thus folders):

1. Raw: the 12 raw images, registered and radiometrically corrected,

2. Multispectral: the set of 6 -band multispectral images taken at four polarization angles $\left(0^{\circ}, 45^{\circ}\right.$, $90^{\circ}$ and $135^{\circ}$ ), after dual-RGB recombination of bands. 
3. Data: contains the matrices resulting from the spectral and polarimetric calibrations. It is useful for Stokes and reflectance estimations.

The files can be downloaded at https://github.com/pjlapray or by simple request by email to the corresponding author, where a zip file contains directories, one directory for each of the scenes. Figure 6 shows an example of different processed images from the scene called "Painting".

\subsection{Spectral estimation evaluation}

Spectral reflectances estimation is evaluated using the MCCPP. The linear model method from Equation 4 is applied over the camera response values. Figure 7 and 8 shows the estimated and measured (reference) spectral reflectances of the 26 patches of the Creative Enhancement chart in the visible and near-infrared parts of the spectrum respectively. The RMSE (Root Mean Square Error) has been selected as a spectral metric. It is defined by:

$$
R M S E=\sqrt{\frac{1}{n} \sum_{j=1}^{n}\left[\tilde{R}\left(\lambda_{j}\right)-R\left(\lambda_{j}\right)\right]^{2}}
$$

From the spectral plots, we see that the estimations are very close to the ground truth in the visible part of the spectrum $(420-720 \mathrm{~nm})$. It is fairly good in the near-infrared parts of the spectrum when reflectance is near to flat $(720-1000 \mathrm{~nm})$. But it fails in the near-infrared spectrum when spectral behavior of patches are varying widely. It is due to the large spectral gap that exists between the channel 5 and 6 (see Figure 4(d)) of the acquisition system.

\subsection{Discussion}

There are several aspects that we identified, and that could potentially be investigated in the future.

First, the spectral bands of dual-RGB cameras appear to be inequitably balanced in terms of energy. Thus, noise affects inequitably the resulting multispectral images. Correction could be done by filter design consideration ${ }^{32}$ or by a pre-processing imaging pipeline using High Dynamic Range imaging. ${ }^{34,35}$ Moreover, several state-of-the-art works handle with optimal filter selection to get a better reflectance estimation. ${ }^{36}$ This selection is done using specific bandpass filters from a set of available manufactured filters. In our case, we took the same bandpass filters as in Berns et al., ${ }^{28}$ because we found that the resulting sensitivities are well uniformly spaced (see Figure 2).

Secondly, drawbacks on spectral estimation errors have to be considered for all materials. In this work, reflectance estimation is done using training data that is not very representative of all natural and man-made surface of objects tested. More robust spectral calibration using a more evolved method and more materials could be employed for a better estimation.

A significant polarized component exists from the objects due to the incident angle of light $\left(45^{\circ}\right)$. It appears that a lot of highlights could be removed using polarization processing. Fully polarization filtered images $\left(\hat{\boldsymbol{U}}_{\boldsymbol{i}}\right)$ can be produced by the following equation:

$$
\hat{\boldsymbol{U}}_{\boldsymbol{i}}=\hat{s}_{0, i}-\sqrt{\left(\hat{s}_{1, i}\right)^{2}+\left(\hat{s}_{2, i}\right)^{2}}
$$

Figure $6(t)$ is a sRGB representation of data using this polarization component removing. We can see that a lot of specular polarized highlights are removed. Thus, it would be interesting to evaluate reflectance reconstruction and calculate RMSE using different kind of data from the pipeline: light intensity $\left(\hat{\boldsymbol{S}}_{\boldsymbol{i}}\right)$, single-angle polarization filtered $\left(\boldsymbol{\rho}_{\boldsymbol{i}}\right)$, and fully polarization filtered $\left(\hat{\boldsymbol{U}}_{\boldsymbol{i}}\right)$. This study will be done in the future.

Dynamic range of values is an important parameter in polarimetric acquisition, because quality of polarimetric descriptors are very sensitive to noise in images acquired. ${ }^{37}$ Moreover, scenes containing specular highlights needs to capture a wide range of intensities. Well-known High Dynamic Range (HDR) 
technique $^{38,39}$ could be employed to resolve these issues. Taking multiple exposure times of the same scene, and combine them into a relative radiance map seems to be a valid method. In order to not increase the complexity of the imaging acquisition pipeline, we preferred not to use such method, but evaluation of polarimetric and multispectral image quality using HDR will be considered as a future work.

We are aware that our imaging technique is not suitable for practical imaging in outdoor environment, as it is relatively slow for capturing data. Real-time achievements of hybrid spectral/polarization capture is at his very beginning, and we believe that investigating dedicated imaging pipeline for these future device is an important subject of research. Our goal was to investigate an imaging pipeline, as well as providing some real data to the community, thus snapshot imaging is off topic here.

\section{CONCLUSION}

Joint spectral and polarimetric capture were investigated in this work. We define an imaging model that permits to recover both spectral reflectance and Stokes estimations. We developed an experimental setup that implements the imaging pipeline, and provides data calibration and image dataset.

The data presented in this work allow to analyze, for example, the degree and angle of polarization for each spectral band. Further analysis would be interesting to study the correlations between spectral and polarization signatures of different material in the visible and near-infrared range.

\section{ACKNOWLEDGMENTS}

We want to specially thanks Dr. Yusuke Monno and his team for providing the spectral measurements of the ColorChecker Passport.

\section{REFERENCES}

[1] Horváth, G. and Varjú, D., [Polarized light in animal vision: polarization patterns in nature], Springer Science \& Business Media (2013).

[2] Nayar, S. K., Fang, X.-S., and Boult, T., "Separation of reflection components using color and polarization," International Journal of Computer Vision 21, 163-186 (Feb 1997).

[3] Goudail, F., Terrier, P., Takakura, Y., Bigué, L., Galland, F., and DeVlaminck, V., "Target detection with a liquid-crystal-based passive stokes polarimeter," Appl. Opt. 43, 274-282 (Jan 2004).

[4] Atkinson, G. A. and Hancock, E. R., "Shape estimation using polarization and shading from two views," IEEE Transactions on Pattern Analysis and Machine Intelligence 29, 2001-2017 (Nov 2007).

[5] Wolff, L. B., "Polarization-based material classification from specular reflection," IEEE Transactions on Pattern Analysis and Machine Intelligence 12, 1059-1071 (Nov 1990).

[6] Roy S. Berns, F. H. I. and Peter D. Burns, D.-Y. T., "Multispectral-based color reproduction research at the munsell color science laboratory," Proc.SPIE 3409, 3409 - 3409 - 12 (1998).

[7] Benezeth, Y., Sidibé, D., and Thomas, J.-B., "Background subtraction with multispectral video sequences," in [IEEE International Conference on Robotics and Automation workshop on Nonclassical Cameras, Camera Networks and Omnidirectional Vision (OMNIVIS)], 6-p (2014).

[8] Rfenacht, D., Fredembach, C., and Süsstrunk, S., "Automatic and accurate shadow detection using near-infrared information," IEEE Transactions on Pattern Analysis and Machine Intelligence 36, 1672-1678 (Aug 2014).

[9] Thomas, J. B., "Illuminant estimation from uncalibrated multispectral images," in [2015 Colour and Visual Computing Symposium (CVCS)], 1-6 (Aug 2015).

[10] Lapray, P.-J., Wang, X., Thomas, J.-B., and Gouton, P., "Multispectral filter arrays: Recent advances and practical implementation," Sensors 14(11), 21626-21659 (2014).

[11] Wang, X., Thomas, J.-B., Hardeberg, J. Y., and Gouton, P., "Multispectral imaging: narrow or wide band filters?," JAIC-Journal of the International Colour Association 12 (2014). 
[12] Alsam, A., Connah, D., and Hardeberg, J., "Multispectral imaging: How many sensors do we need?," Journal of Imaging Science and Technology 50(1), 45-52 (2006).

[13] Schaul, L., Fredembach, C., and Süsstrunk, S., "Color image dehazing using the near-infrared," in [2009 16th IEEE International Conference on Image Processing (ICIP)], 1629-1632 (Nov 2009).

[14] Salamati, N., Larlus, D., Csurka, G., and Süsstrunk, S., "Semantic image segmentation using visible and near-infrared channels," in [European Conference on Computer Vision], 461-471, Springer (2012).

[15] Yan, Q., Shen, X., Xu, L., Zhuo, S., Zhang, X., Shen, L., and Jia, J., "Cross-field joint image restoration via scale map," in [2013 IEEE International Conference on Computer Vision], 15371544 (Dec 2013).

[16] Sabatke, D. S., Locke, A. M., Dereniak, E. L., Descour, M. R., Garcia, J. P., Hamilton, T. K., and McMillan, R. W., "Snapshot imaging spectropolarimeter," Optical engineering 41(5), 1048-1055 (2002).

[17] Alouini, M., Goudail, F., Grisard, A., Bourderionnet, J., Dolfi, D., Bénière, A., Baarstad, I., Løke, T., Kaspersen, P., Normandin, X., and Berginc, G., "Near-infrared active polarimetric and multispectral laboratory demonstrator for target detection," Appl. Opt. 48, 1610-1618 (Mar 2009).

[18] Gruev, V., "Bio-inspired spectral-polarization imaging sensors for medical applications," in [Frontiers in Optics 2014], Frontiers in Optics 2014, FW2E.2, Optical Society of America (2014).

[19] Mu, T., Pacheco, S., Chen, Z., Zhang, C., and Liang, R., "Snapshot linear-stokes imaging spectropolarimeter using division-of-focal-plane polarimetry and integral field spectroscopy," Scientific Reports 7, 42115 (2017).

[20] Garcia, M., Edmiston, C., Marinov, R., Vail, A., and Gruev, V., "Bio-inspired color-polarization imager for real-time in situ imaging," Optica 4, 1263-1271 (Oct 2017).

[21] Lenaic Le Hors, Pierre Hartemann, S. B., "Multispectral polarization active imager in the visible band," Proc.SPIE 4035, 4035 - 4035 - 10 (2000).

[22] Shen, H.-L., Cai, P.-Q., Shao, S.-J., and Xin, J. H., "Reflectance reconstruction for multispectral imaging by adaptive wiener estimation," Opt. Express 15, 15545-15554 (Nov 2007).

[23] Maloney, L. T., "Evaluation of linear models of surface spectral reflectance with small numbers of parameters," J. Opt. Soc. Am. A 3, 1673-1683 (Oct 1986).

[24] Imai, F. H. and Berns, R. S., "Spectral estimation using trichromatic digital cameras," in [Proceedings of the International Symposium on Multispectral Imaging and Color Reproduction for Digital Archives], 42, 1-8, Chiba University Chiba, Japan (1999).

[25] David R. Connah, J. Y. H., "Spectral recovery using polynomial models," Proc.SPIE 5667, 5667 5667 - 11 (2005).

[26] Zhao, Y. and Berns, R. S., "Image-based spectral reflectance reconstruction using the matrix $\mathrm{r}$ method," Color Research \& Application 32(5), 343-351 (2007).

[27] Myhre, G., Hsu, W.-L., Peinado, A., LaCasse, C., Brock, N., Chipman, R. A., and Pau, S., "Liquid crystal polymer full-stokes division of focal plane polarimeter," Opt. Express 20, 27393-27409 (Dec 2012).

[28] Berns, R. S., Taplin, L. A., Nezamabadi, M., Zhao, Y., and Mohammadi, M., "Practical spectral imaging using a color-filter array digital camera," Studies in Conservation (2006).

[29] Evangelidis, G., "Iat: A matlab toolbox for image alignment." http://www.iatool.net (2013).

[30] Persons, C. M., Jones, M. W., Farlow, C. A., Morell, L. D., Gulley, M. G., and Spradley, K. D., "A proposed standard method for polarimetric calibration and calibration verification," in [Polarization Science and Remote Sensing III], 6682, 66820K, International Society for Optics and Photonics (2007).

[31] Westland, S., Ripamonti, C., and Cheung, V., [Computational colour science using MATLAB], John Wiley \& Sons (2012).

[32] Lapray, P.-J., Thomas, J.-B., Gouton, P., and Ruichek, Y., "Energy balance in spectral filter array camera design," Journal of the European Optical Society-Rapid Publications 13, 1 (Jan 2017). 
[33] Monno, Y., Kikuchi, S., Tanaka, M., and Okutomi, M., "A practical one-shot multispectral imaging system using a single image sensor," IEEE Transactions on Image Processing 24, 3048-3059 (Oct 2015).

[34] Lapray, P.-J., Thomas, J.-B., and Gouton, P., "High dynamic range spectral imaging pipeline for multispectral filter array cameras," Sensors 17(6) (2017).

[35] Lapray, P. J., Heyrman, B., Rossé, M., and Ginhac, D., "Smart camera design for realtime high dynamic range imaging," in [2011 Fifth ACM/IEEE International Conference on Distributed Smart Cameras], 1-7 (Aug 2011).

[36] Vrhel, M. J. and Trussell, H. J., "Optimal scanning filters using spectral reflectance information," in [Human Vision, Visual Processing, and Digital Display IV], 1913, 404-413, International Society for Optics and Photonics (1993).

[37] Alouini, M., Goudail, F., Refregier, P., Grisard, A., Lallier, E., and Dolfi, D., "Multispectral polarimetric imaging with coherent illumination: towards higher image contrast," in [Proceedings of SPIE], 5432, 133-144 (2004).

[38] Debevec, P. E. and Malik, J., "Recovering high dynamic range radiance maps from photographs," in [Proceedings of the 24th Annual Conference on Computer Graphics and Interactive Techniques], SIGGRAPH '97, 369-378, ACM Press/Addison-Wesley Publishing Co., New York, NY, USA (1997).

[39] Lapray, P. J., Heyrman, B., Rossé, M., and Ginhac, D., "Hdr-artist: High dynamic range advanced real-time imaging system," in [2012 IEEE International Symposium on Circuits and Systems], 14281431 (May 2012). 


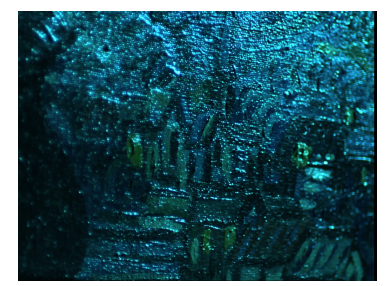

(a) $0^{\circ}$

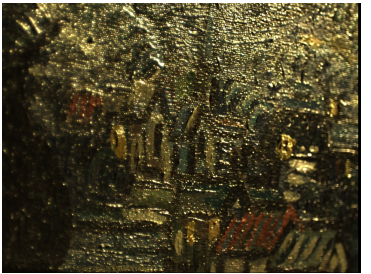

(e) $0^{\circ}$

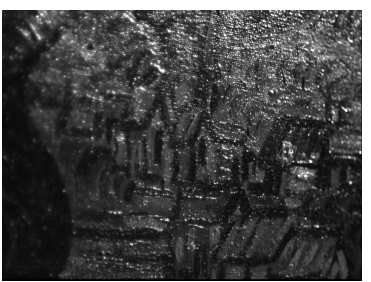

(i) $0^{\circ}$

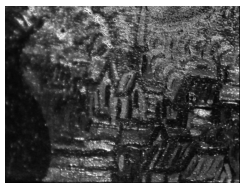

(m) $\hat{s}_{0}$ band 1

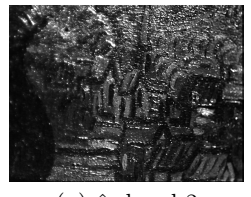

(n) $\hat{s}_{0}$ band 2

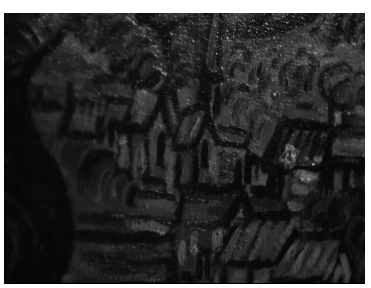

(j) $45^{\circ}$

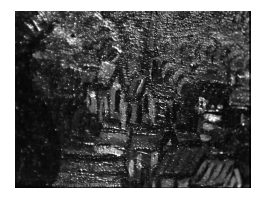

(o) $\hat{s}_{0}$ band 3

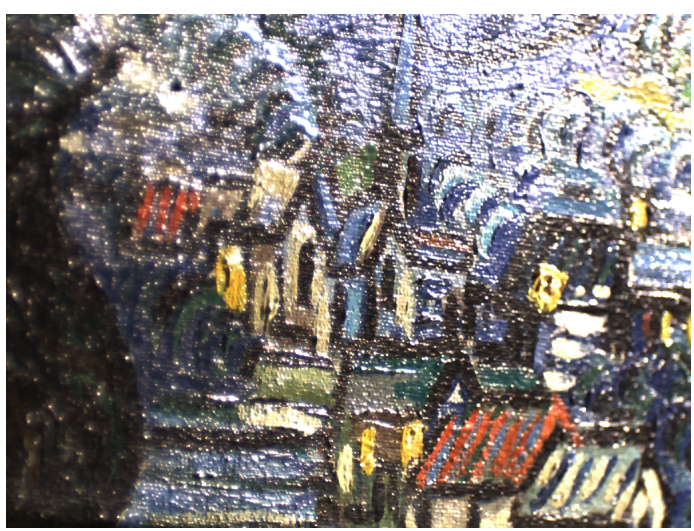

(s) Simple RGB

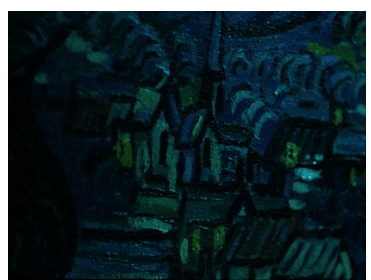

(c) $90^{\circ}$

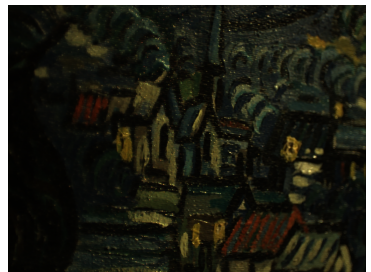

(g) $90^{\circ}$

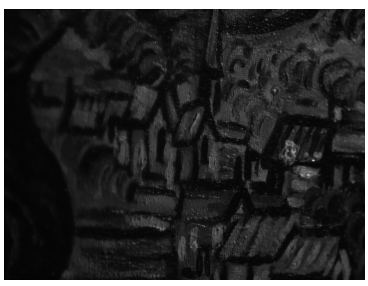

(k) $90^{\circ}$

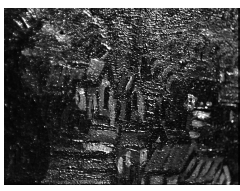

(p) $\hat{s}_{0}$ band 4

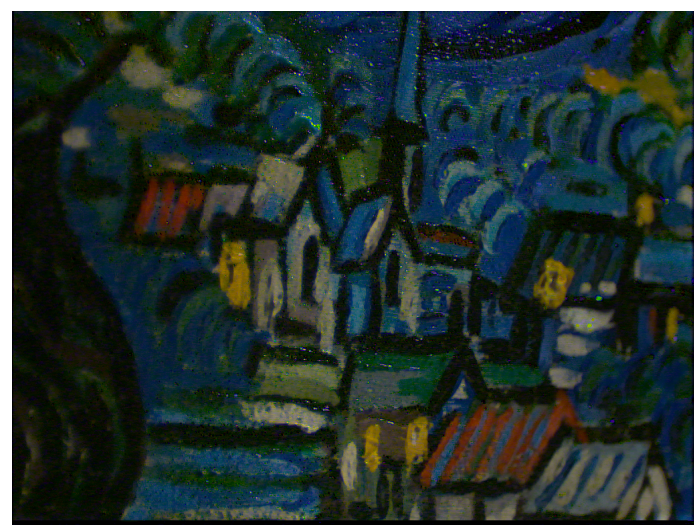

(t) sRGB transform after polarization component removing

Figure 6. Visualization of the painting scene. (a)-(d) Raw images through the blue-green filter. (e)-(h) Raw images through the yellow filter. (i)-(l) Near-infrared raw images. (m)-(r) Multispectral $\hat{s}_{0, i}$ images reconstructed from the dual-rgb method and using the pipeline described in Figure 1. (s) Simple color image taken from the RGB sensor without any filters. (t) sRGB visualization from a linear transform of multispectral images to sRGB, after removing the polarization component. 


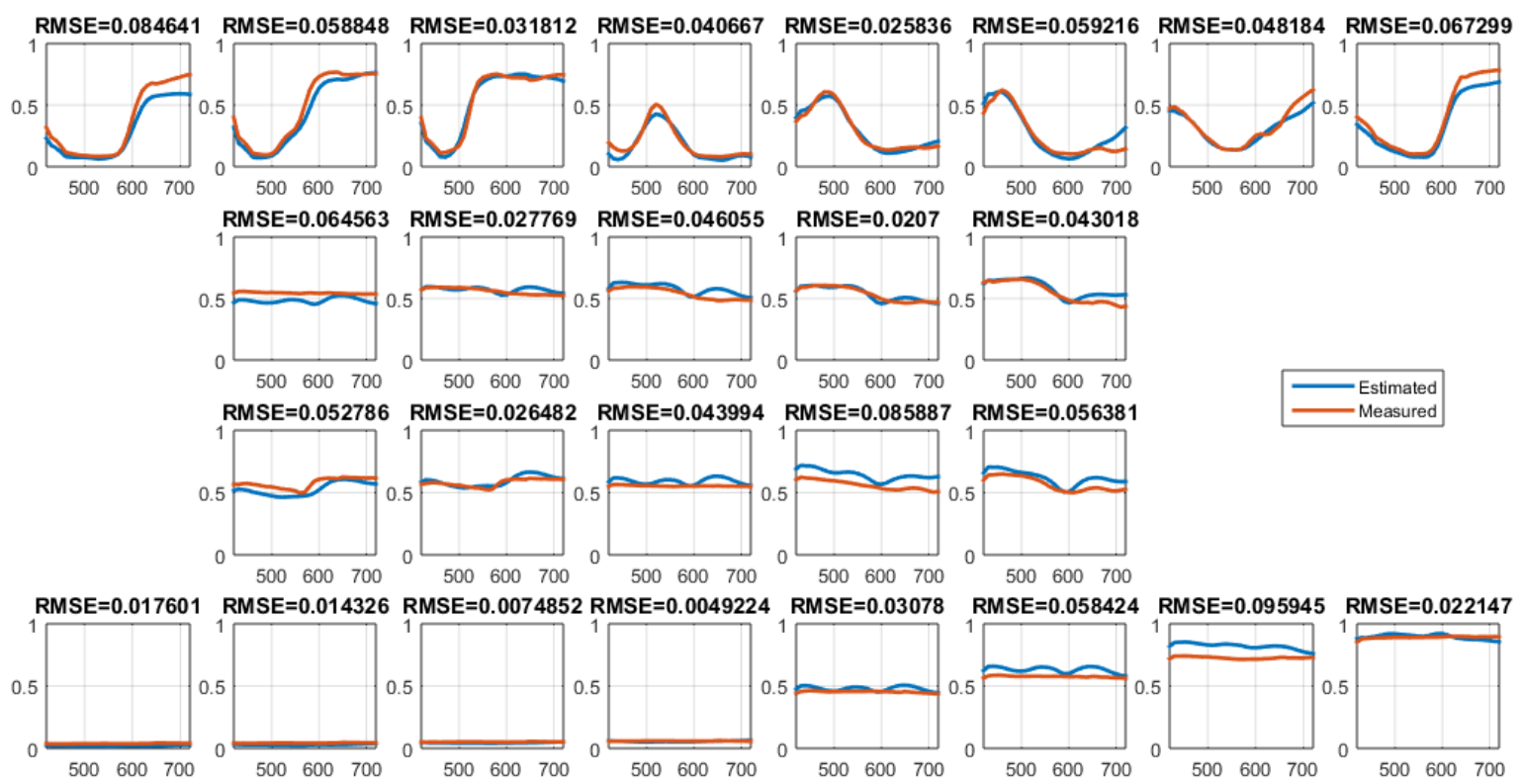

Figure 7. Measured and estimated spectral reflectance of the creative enhancement chart in the visible part of the spectrum $(420-720 \mathrm{~nm})$. Measured data is from a spectrometer and is used as a reference.
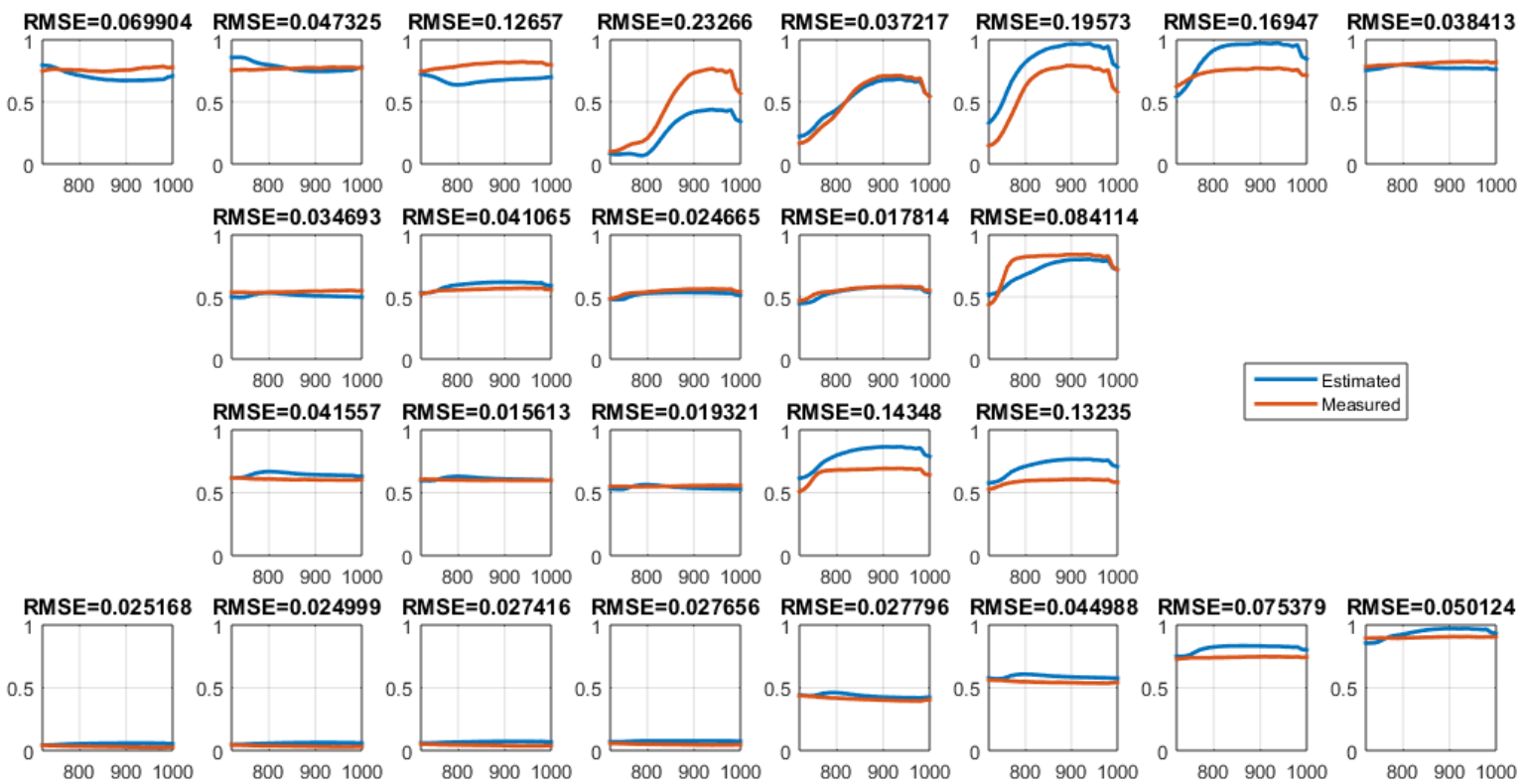

Figure 8. Measured and estimated spectral reflectance of the creative enhancement chart in the near-infrared part of the spectrum $(720-1000 \mathrm{~nm})$. Measured data is from a spectrometer and is used as a reference. 